\title{
APPLICATION OF LIAPUNOV'S DIRECT METHOD TO FIXED POINT THEOREMS
}

\author{
J. H. GEORGE, ${ }^{1}$ V. M. SEHGAL AND R. E. SMITHSON
}

Abstract. The direct method of Liapunov is applied to the existence of fixed points for multivalued functions. Many recent fixed point theorems are shown to be special cases of the Liapunov theory.

1. Introduction and definitions. Liapunov's direct method has been one of the major tools in solving many problems of ordinary differential equations. For example, Yoshizawa [9] has used this method to obtain necessary and sufficient conditions for the continuability of solutions of ordinary differential equations. The purpose of this paper is to use Liapunov theory to prove certain fixed point theorems for multivalued functions.

Further we shall obtain as corollaries to the main theorem the results of Belluce and Kirk [1], Browder and Petryshyn [2], Diaz and Metcalf [3], Edelstein [4], Nadler [5], and others.

Let $(X, d)$ be a metric space and let $F: X \rightarrow X$ be a multifunction on $X$ into $X$. (A multifunction is a correspondence on $X$ such that $F(x)$ is a subset of $X$ for each $x \in X$.) We shall use the word function in case $F$ is single valued and we shall usually use $f$ to denote the function. In the following $F$ will denote a multifunction on the metric space $X$ into $X$.

Definitions. (1) The multifunction $F$ is point closed (compact) if and only if $F(x)$ is closed (compact) for each $x \in X$.

(2) The multifunction $F$ is upper semicontinuous (u.s.c.) if and only if whenever $F(x) \subset V$, an open set, then there is an open set $U$, containing $x$, such that $F(y) \subset V$ for all $y \in U$.

(3) The multifunction $F$ is lower semicontinuous (1.s.c.) if and only if $F(x) \cap V \neq \varnothing$, where $V$ is an open set, implies that there is an open set $U$ containing $x$ such that $F(z) \cap V \neq \varnothing$ for all $z \in U$.

(4) Finally $F$ is continuous if and only if it is both u.s.c. and l.s.c.

If $A \subset X$, let $F^{-1}(A)=\{x: F(x) \cap A \neq \varnothing\}$, and $F(A)$ $=\bigcup\{F(x): x \in A\}$. Further, $\operatorname{cl}(A)$ denotes the closure of $A$, and $A^{0}$ the interior.

Received by the editors May 3, 1970.

AMS 1969 subject classifications. Primary 5465, 5485.

Key words and phrases. Contractive multivalued functions, fixed point theorems, orbits for multifunctions, cluster points of orbits, Liapunov theory.

1 This author was partially supported by NAS 8-21434. 
We extend the notion of the orbit of a point in the following way. Let $x_{0} \in X$. Then an orbit of $x_{0}$ is a sequence $\left\{x_{n}, n \in W\right.$, the positive integers $\}$ such that $x_{n} \in F\left(x_{n-1}\right)$ for $n \geqq 1$. Note that, since we are considering multifunctions, a point may have many orbits. We denote an orbit of $x$ by $\mathcal{O}(x)$, and we also use this symbol to denote the range of the sequence.

Definitions. (1) The multifunction $F$ is sequentially continuous on an orbit $\mathcal{O}(x)$ if and only if there is a convergent subsequence $x_{n_{i}} \rightarrow y_{0}$ of $\mathcal{O}(x)$, such that for each $k \in W$, there is an element $y_{k} \in F\left(y_{k-1}\right)$ such that $x_{n_{i}+k} \rightarrow y_{k}$.

(2) Also we say that $F$ satisfies a Lipschitz condition if and only if there is a real number $\alpha \geqq 0$ such that for $x, y \in X$, and $w \in F(x)$, there is a $z \in F(y)$ such that $d(w, z) \leqq \alpha d(x, y)$.

Note that each continuous single valued function is sequentially continuous on each orbit which contains a convergent subsequence.

The proof of the following lemmas can be constructed directly from the definitions and are omitted. (See Smithson [6] or Strother [8] for basic results on multifunctions.)

Leмma 1.1. Let $F$ be an upper semicontinuous, point closed multifunction. If $x_{n} \rightarrow x_{0}$, and $y_{n} \rightarrow y_{0}$ where $y_{n} \in F\left(x_{n}\right)$, then $y_{0} \in F\left(x_{0}\right)$.

Lемма 1.2. If $F$ is point compact and satisfies a Lipschitz condition, then $F$ is continuous.

LеммA 1.3. If $F$ is point compact and u.s.c., if $x_{n} \rightarrow x_{0}$ and if $y_{n} \in F\left(x_{n}\right)$ for each $n$, then there is a $y_{0} \in F\left(x_{0}\right)$ and a subsequence $y_{n_{i}} \rightarrow y_{0}$.

REMARKs. (1) Let $F$ be point compact and u.s.c. Then if $\theta(x)$ contains a convergent subsequence we can construct a subsequence $x_{n_{i}} \rightarrow y_{0}$ of $\mathcal{O}(x)$ such that $x_{n_{i}+1} \rightarrow y_{1} \in F\left(y_{0}\right)$ and inductively such that $x_{n_{i}+k} \rightarrow y_{k} \in F\left(y_{k-1}\right)$.

(2) By applying a diagonal process to the results in Remark 1, we can show that any point compact u.s.c. multifunction is sequentially continuous on each orbit which contains a convergent subsequence. Further, the same kind of reasoning will establish the following lemma.

LEMMA 1.4. If $F$ is l.s.c. and $\mathcal{O}(x)$ contains a convergent subsequence, or if $F$ is u.s.c. point closed, and $X$ is compact, then $F$ is sequentially continuous.

Definition. Let $\Theta(x)$ be an orbit of $x$. Then a Liapunov function for $F$ on $\Theta(x)$ is a continuous single valued function $V: X \rightarrow R$ such that 
(i) $V\left(x_{n+1}\right) \leqq V\left(x_{n}\right)$ for all $x_{n} \in \mathcal{O}(x)$ and

(ii) $V$ is bounded below.

REMARK. For many of our results we need only define $V$ on $\operatorname{cl}(\mathcal{O}(x))$.

As an example of a Liapunov function, let $X=[0,1]$ and let $0<\alpha<1$. Then define $F(x)=[0, \alpha x]$, and for $x_{0} \in X$, let $\mathcal{O}\left(x_{0}\right)$ $=\left\{x_{n}: x_{n}=\alpha x_{n-1}=\alpha^{n} x_{0}, n \geqq 1\right\}$. Then set $V(x)=x$; then $V$ will be a Liapunov function for $F$ on $\mathcal{O}(x)$.

Finally a fixed point of $F$ is a point such that $x \in F(x)$.

2. The main theorem. The main theorem of this paper depends on the following fundamental property of Liapunov functions.

Lemma 2.1. Let $V$ be a Liapunov function on the orbit $\mathcal{O}(x)$. If there is a convergent subsequence $x_{n_{i}} \rightarrow y_{0}$ of $\mathcal{O}(x)$ such that $x_{n_{i}+1} \rightarrow y_{1}$, then $V\left(y_{0}\right)=V\left(y_{1}\right)$, and if $x_{n_{i}+k} \rightarrow y_{k}$, then $V\left(y_{0}\right)=V\left(y_{k}\right)$.

Proof. Since $x_{n_{i}} \rightarrow y_{0}$ and since $V$ is continuous, $V\left(x_{n_{i}}\right) \rightarrow V\left(y_{0}\right)$ and $V\left(x_{n_{i}+1}\right) \rightarrow V\left(y_{1}\right)$. Furthermore, $V\left(x_{n_{i}+1}\right) \leqq V\left(x_{n_{i}}\right)$ and therefore $V\left(y_{1}\right)$ $\leqq V\left(y_{0}\right)$. On the other hand, $V\left(x_{n_{j}}\right) \leqq V\left(x_{n_{i}+1}\right)$ for $j>i$, and so $V\left(y_{0}\right) \leqq V\left(y_{1}\right)$. The final statement follows by induction.

TheOREM 2.2. Let $F: X \rightarrow X$ and let $\mathcal{O}(x)$ be an orbit for $F$ at $x$ with convergent subsequences $x_{n_{i}} \rightarrow y_{0}$ and $x_{n_{i}+k} \rightarrow y_{k}$ for $k=1, \cdots, m$. If there is a Liapunov function $V$ on $\mathcal{O}(x)$ such that $y_{0} \neq y_{k}$ implies that $V\left(y_{0}\right) \neq V\left(y_{k}\right)$ for some $k, 1 \leqq k \leqq m$, then $y_{0}=y_{k}$. Further, if $k=1$ and $y_{\mathrm{i}} \in F\left(y_{0}\right)$, then $y_{0}$ is a fixed point.

Proof. By Lemma 2.1, $V\left(y_{0}\right)=V\left(y_{k}\right)$ and hence, the hypothesis implies that $y_{0}=y_{1}$.

At first Theorem 2.2 appears to be trivial. However, the consequences of this theorem are many of the well-known fixed point theorems as we will show later. The key point is of course the existence of the Liapunov function $V$ which distinguishes limit points. In the sequel we shall obtain a number of results by constructing such a Liapunov function. A major result in this direction is:

Lemma 2.3. If $F: X \rightarrow X$ is continuous and point compact, then $V(x)=d(x, F(x))$ defines a continuous function on $X$ into $R$.

Proof. Note that since $F(x)$ is compact, there is a $y \in F(x)$ such that $d(x, y)=d(x, F(x))$. Then suppose that $x_{n} \rightarrow x_{0}$, and let $y_{n} \in F\left(x_{n}\right)$ such that $d\left(x_{n}, y_{n}\right)=d\left(x_{n}, F\left(x_{n}\right)\right)$. Since $F$ is u.s.c. and point compact, some subsequence $y_{n_{i}} \rightarrow y_{0}$ converges to a point in $F\left(x_{0}\right)$. Then if $d\left(x_{0}, F\left(x_{0}\right)\right)=d\left(x_{0}, y_{0}\right)$, the continuity of the metric $d$ implies that $V\left(x_{n}\right) \rightarrow V\left(x_{0}\right)$. Thus suppose there is a $y^{\prime} \in F\left(x_{0}\right)$ such that $d\left(x_{0}, y^{\prime}\right)$ 
$<d\left(x_{0}, y_{0}\right)$, and set $\epsilon=\left(d\left(x_{0}, y_{0}\right)-d\left(x_{0}, y^{\prime}\right)\right) / 2$. By applying the 1.s.c. of $F$, we can find an $n$ and a $z_{n} \in F\left(x_{n}\right)$ such that $d\left(y^{\prime}, z_{n}\right)<\epsilon$. But this implies

$$
d\left(x_{n}, F\left(x_{n}\right)\right) \leqq d\left(x_{n}, z_{n}\right)<d\left(x_{n}, y_{n}\right)=d\left(x_{n}, F\left(x_{n}\right)\right)
$$

which is a contradiction. Hence, $V\left(x_{n}\right) \rightarrow V\left(x_{0}\right)$ and $V$ is continuous.

As examples of the applicability of Theorem 2.2 we present the following corollaries. Corollary 2.5 is a result of Nadler [5].

CoRollary 2.4. Let $F: X \rightarrow X$ be a continuous point compact multifunction on $X$. Let $\mathcal{O}(x)$ be an orbit for $F$ which contains a convergent subsequence. If the function $V$ defined by $V(x)=d(x, F(x))$ is decreasing on $\mathcal{O}(x)$, and if $V\left(x_{n}\right) \rightarrow 0$, then $F$ has a fixed point.

Proof. By hypothesis and Lemma 2.3, $V$ is a Liapunov function on $\mathcal{O}(x)$. Further, if $x_{n_{i}} \rightarrow y_{0}$, we may assume $x_{n_{i}+1} \rightarrow y_{1} \in F\left(y_{0}\right)$ by the remark following Lemma 1.3. Then the result follows from Theorem 2.2 .

Corollary 2.5. If $X$ is compact, if $F$ is point closed and satisfies a Lipschitz condition with $\alpha<1$, then $F$ has a fixed point.

Proof. Construct $\mathcal{O}(x)$ by choosing $x_{n} \in F\left(x_{n-1}\right)$ so that $d\left(x_{n}, x_{n-1}\right)$ $=d\left(x_{n-1}, F\left(x_{n-1}\right)\right)$. Then the Lipschitz condition shows that $V$ is decreasing on $\mathcal{O}(x)$, and if $x_{n_{i}} \rightarrow y_{0}, x_{n_{i}+1} \rightarrow y_{1}$, then $y_{0} \neq y_{1}$ implies $V\left(y_{0}\right) \neq V\left(y_{1}\right)$. Hence, $F$ has a fixed point.

REMARK. If $F$ in Corollary 2.5 is single valued, then we have a variation on the classic theorem on contraction mappings. In this case we would obtain a unique fixed point. However, we cannot obtain a unique fixed point for multifunctions. For if $F(x)=X$ for all $x \in X$, then $F$ satisfies a Lipschitz condition with any constant $\alpha>0$. But every point in $X$ is a fixed point. Finally the assumption that $X$ is compact in Corollary 2.5 can be replaced by $X$ complete.

3. Applications of the main theorem. In this section we obtain generalizations of several well-known fixed point theorems for single valued functions.

Definitions. The multifunction $F: X \rightarrow X$ is:

(1) contractive iff for all $x \neq y \in X$ and for each $z \in F(x)$ there exists a $w \in F(y)$ such that $d(w, z)<d(x, y)$;

(2) $\epsilon$-locally contractive iff for all $x \neq y \in X$ with $d(x, y)<\epsilon$ and for all $z \in F(x)$, there is a $w \in F(y)$ such that $d(z, w)<d(x, y)$;

(3) nonexpansive iff $F$ satisfies a Lipschitz condition with $\alpha=1$;

(4) said to have diminishing orbital diameters iff whenever each orbit of $x$ has positive diameter, then 


$$
\lim _{n} \delta\left\{x_{k}: k \geqq n\right\}<\inf \{\delta(\mathcal{O}(x)): \text { for each } \mathcal{O}(x)\}
$$

where the terms $x_{k}$ are the terms of a particular orbit and where $\delta(A)$ denotes the diameter of the set $A$.

We state without proof the following lemma.

Lемма 3.1. If $F$ satisfies any of (1), (2), or (3) and if $F$ is point compact, then $F$ is continuous. Hence, $V(x)=d(x, F(x))$ defines a continuous function on $X$.

In the following $V$ will denote the above function unless otherwise stated.

Definition. An orbit $\mathcal{O}(x)$ is called regular iff $d\left(x_{n}, x_{n+1}\right)$ $=d\left(x_{n}, F\left(x_{n}\right)\right)$ for all $n$.

REMARK. If $F$ is nonexpansive and point compact, then the function $V$ is decreasing on regular orbits, and hence, is a Liapunov function.

The next theorem is essentially a theorem of Smithson [7] which is a generalization of a theorem of Edelstein [4].

THEOREM 3.2. If $F: X \rightarrow X$ is a contractive, point compact multifunction, and if there is a regular orbit for $F$ which contains a convergent subsequence, then $F$ has a fixed point.

Proof. Let $\mathcal{O}(x)$ be a regular orbit, and suppose that $x_{n_{i}} \rightarrow y_{0}$, and $x_{n_{i}+1} \rightarrow y_{1} \in F\left(y_{0}\right)$ (see the remark following Lemma 1.3). By Lemma 3.1 , and the remark, $V$ is a Liapunov function on $\mathcal{O}(x)$. Finally, if $y_{0} \neq y_{1}$ then, since $F$ is contractive, $V\left(y_{1}\right)<V\left(y_{0}\right)$ (see the proof of Lemma 2.1). Thus $y_{0}=y_{1}$ and $F$ has a fixed point.

Corollary 3.3. If $F: X \rightarrow X$ is a contractive, point closed multifunction on the compact space $X$, then $F$ has a fixed point.

Another corollary to Theorem 3.2 is easily obtained, but first we need the following definition.

Definition. A single valued function $f: X \rightarrow X$ is almost periodic at $p \in X$ iff for each $\epsilon>0$ there is an $n$ such that $d\left(f^{n}(p), p\right)<\epsilon$.

Corollary 3.4. If $f: X \rightarrow X$ is a contractive function which is almost periodic at $p$, then $f(p)=p$.

We also obtain the following result on periodic points of $\epsilon$-locally contractive mappings.

THEOREM 3.5. Let $F$ be an e-locally contractive, point compact multifunction on $X$. If $\mathcal{O}(x)$ is a regular orbit which contains a convergent 
subsequence, and if $F$ is sequentially continuous on $\mathcal{O}(x)$, then there is a positive integer $m$ such that $F^{m}$ has a fixed point.

Proof. Let $x_{n_{i}} \rightarrow y_{0}$ be a convergent subsequence of $\mathcal{O}(x)$, and let $x_{n_{i}+k} \rightarrow y_{k}$ where $y_{k} \in F\left(y_{k-1}\right)$ for each $k \geqq 1$. Pick $N$ such that $d\left(x_{n_{i}}, x_{n_{j}}\right)$ $\langle\epsilon / 4$ for all $i, j>N$. Then since $\theta(x)$ is regular and $F$ is an $\epsilon$-locally contractive multifunction, $d\left(x_{n_{i}+k}, x_{n_{j}+k}\right)<\epsilon / 4$ for $k \geqq 0$. Fix $i>N$ and set $m=n_{i+1}-n_{i}$. Then $n_{i}+m=n_{i+1}$ and thus $d\left(x_{n_{i}+m}, x_{n_{i}}\right)<\epsilon / 4$. By combining these inequalities we deduce that $d\left(y_{0}, y_{m}\right)<\epsilon$.

Now let $Y=\left\{x_{n_{i}+l}: l=k m, k \geqq 0\right\} \cup\left\{y_{j}: j=k m, k \geqq 0\right\}$, and define $g: Y \rightarrow Y$ by $g\left(x_{n_{j}}\right)=x_{n_{j}+m}$ and $g\left(y_{j}\right)=y_{j+m}$. Then $g$ is an $\epsilon$-locally contractive function on $Y$ into $Y$ and $V(z)=d(z, g(z))$ defines a Liapunov function for $g$ on $\mathcal{O}\left(x_{n_{i}}\right)$ in $Y$. Furthermore, if $y_{0} \neq y_{m}$, then $V\left(y_{0}\right)$ $\neq V\left(y_{m}\right)$. Thus Theorem 2.2 implies that $y_{0}=y_{m}$ and hence, $y_{0} \in F^{m}\left(y_{0}\right)$.

LеммA 3.6. Let $F$ be a nonexpansive function. If there is an $x \in X$ which has an orbit with finite diameter, then there is an orbit with finite diameter for every $z \in X$, and the function

$$
V(z)=\inf \{\delta(\Theta(z)): \Theta(z) \text { an orbit of } z\}
$$

is uniformly continuous.

Proof. Suppose that $\mathcal{O}(x)$ has finite diameter, and let $z \in X$. Pick $z_{1} \in F(z)$ such that $d\left(x_{1}, z_{1}\right) \leqq d(x, z)$, and inductively pick $z_{n} \in F\left(z_{n-1}\right)$ such that $d\left(x_{n}, z_{n}\right) \leqq d\left(x_{n-1}, z_{n-1}\right)$. Then

$$
d\left(z_{n}, z_{m}\right) \leqq d\left(z_{n}, x_{n}\right)+d\left(x_{n}, z_{m}\right) \leqq d\left(z_{n}, x_{n}\right)+d\left(x_{n}, x_{m}\right)+d\left(x_{m}, z_{m}\right),
$$

and both statements follow from this inequality.

We now can obtain the following theorem of Belluce and Kirk [1].

THEOREM 3.7. Let $F$ be a nonexpansive multifunction with diminish. ing orbital diameters. If there is an orbit, $\mathcal{O}(x)$, which contains a convergent subsequence on which $F$ is sequentially continuous, then $F$ has a fixed point.

Proof. Let $x_{n_{i}} \rightarrow y$ be a convergent subsequence of $\theta(x)$ and let $x_{n_{i}+k} \rightarrow y_{k}$. Then the function $V$ defined in Lemma 3.6 is a Liapunov function on $\mathcal{O}(x)$, and if $y \notin F(y)$, then $\delta(\mathcal{O}(y))>0$ for all orbits of $y$. Thus there is an $m$ such that $y_{m}$ has an orbit $\mathcal{O}\left(y_{m}\right)$ with

$$
\delta\left(\Theta\left(y_{m}\right)\right)<\inf \{\delta(\Theta(y)): \Theta(y) \text { an orbit of } y\} .
$$

This implies that $V\left(y_{m}\right)<V(y)$ and hence, Theorem 2.2 applies. Thus $y \in F(y)$.

We can obtain a corollary to Theorem 3.7 analogous to the corollary to Theorem 3.2. 
Diaz and Metcalf [3] considered continuous single valued functions for which the set of fixed points was nonempty. Theorems 3.8 and 3.9 are partial generalizations of their results. In the following $\mathcal{F}(F)$ will denote the set of fixed points of a multifunction $F$ and $\mathcal{O}(x)$ will be an orbit of $x$. Then we set $\mathcal{L}(x)$ equal to the set of subsequential limit points of $\mathcal{O}(x)$. We shall also assume that $F$ is sequentially continuous on $\mathcal{O}(x)$.

THEOREM 3.8. If $\mathcal{F}(F) \neq \varnothing$ and compact, and if whenever $z \notin \mathcal{F}(F)$, and $w \in F(z)$, we have $d(w, \mathcal{F}(F))<d(z, \mathcal{F}(F))$, then $\mathcal{L}(x) \subset \mathcal{F}(F)$, and if $\mathfrak{L}(x)=\{y\}$ is a singleton, then $\lim x_{n}=y$.

Proof. For the first statement define $V$ on $\operatorname{cl}(\mathcal{O}(x))$ as follows. If $x_{n} \in \mathcal{O}(x)$, set $V\left(x_{n}\right)=d\left(x_{n}, \mathcal{F}(F)\right)$ and extend $V$ to $\operatorname{cl}(\mathcal{O}(x))$ by taking limits. Then $V$ is a Liapunov function on $\operatorname{cl}(\mathcal{O}(x))$. Let $x_{n_{i}} \rightarrow y$ and $x_{n_{i}+1} \rightarrow y_{1}$. Then if $y \notin F(y), V\left(y_{1}\right)<V(y)$. Thus Theorem 2.2 implies that $y \in F(y)$, that is $\mathcal{L}(x) \subset \mathcal{F}(F)$.

Now suppose that $\mathscr{L}(x)=\{y\}$. Then since $\mathscr{F}(F)$ is compact, for each $x_{n} \in \mathcal{O}(x)$, there is a $z_{n} \in \mathcal{F}(F)$ such that $d\left(x_{n}, \mathfrak{F}(F)\right)=d\left(x_{n}, z_{n}\right)$. Furthermore, by the hypothesis in the theorem, if $m>n$, then $d\left(x_{m}, z_{m}\right)$ $<d\left(x_{n}, z_{n}\right)$. Now suppose that $x_{n_{i}} \rightarrow y$ but that $\Theta(x)$ does not converge to $y$. Then there is an $\epsilon>0$, and a subsequence $\left\{x_{m_{j}}\right\}_{j}$ of $\theta(x)$ such that $d\left(x_{m_{j}}, y\right) \geqq \epsilon$. Let $z$ be a cluster point of the sequence $\left\{z_{m_{j}}\right\}_{j}$. Then, since $d\left(x_{n}, \mathcal{F}(F)\right) \rightarrow 0$, there is a subsequence of $\left\{x_{m_{j}}\right\}_{j}$ which converges to $z$. But $d(z, y) \geqq \epsilon$ which contradicts $\mathcal{L}(x)=\{y\}$.

TheOREM 3.9. If $\mathfrak{F}(F) \neq \varnothing$, and if for each $z \notin \mathcal{F}(F)$, and each $w \in F(z)$, we have $d(w, p)<d(z, p)$ for all $p \in \mathcal{F}(F)$, then either $\mathcal{L}(x)$ is empty or a singleton.

Proof. Let $p$ be a fixed element in $\mathscr{F}(F)$, and set $V(z)=d(z, p)$ for all $z \in X$. Then if $x_{n_{i}} \rightarrow y, V$ is a Liapunov function on $\theta(x)$ which satisfies the conditions of Theorem 2.2. Thus $y \in \mathcal{F}(F)$. Now if $x_{m_{i}} \rightarrow z_{1}$, then $z_{1} \in \mathcal{F}(F)$, and if $\epsilon>0$, there is an $n_{i}$ such that $d\left(y, z_{1}\right) \leqq d\left(x_{n_{i}}, z_{1}\right)+\epsilon$. But if $m_{j}>n_{i}, d\left(x_{m_{j}}, z_{1}\right) \leqq d\left(x_{n_{i}}, z_{1}\right)$ by hypothesis, and hence, $d\left(y, z_{1}\right)$ $=0$. Thus $\mathscr{L}(x)$ is a singleton if $\mathscr{L}(x)$ is nonempty.

Finally, we obtain a generalization of a theorem of Browder and Petryshyn [2] for nonexpansive mappings which are asymptotically regular.

In the following we shall assume that for each $z \in X$, there is an orbit $\mathcal{O}(z)$ such that $d\left(z_{n}, z_{n+1}\right) \rightarrow 0, z_{n}, z_{n+1} \in \mathcal{O}(z)$.

THEOREM 3.10. Let $\mathcal{O}(x)$ be an orbit such that $d\left(x_{n}, x_{n+1}\right) \rightarrow 0$ monotonically and suppose that $F$ is sequentially continuous on $\mathcal{O}(x)$. If $x_{n_{i}} \rightarrow y$, then $y \in F(y)$. 
Proof. Define $V\left(x_{n}\right)=d\left(x_{n}, x_{n+1}\right)$ on $\mathcal{O}(x)$ and extend to $\mathrm{cl}(\mathcal{O}(x))$ by taking limits. Then if $x_{n_{i}+k} \rightarrow y_{k}, V\left(y_{k}\right)=d\left(y_{k}, y_{k+1}\right)$, and $d\left(y_{k}, y_{k+1}\right)$ $\rightarrow 0$. Thus if $y \neq y_{1}$, there is a $k$ such that $V\left(y_{k}\right)<V(y)$, and Theorem 2.2 applies. Thus $y=y_{1}$ and so $y \in F(y)$.

\section{REFERENCES}

1. L. P. Belluce and W. A. Kirk, Fixed-point theorems for certain classes of nonexpansive mapping, Proc. Amer. Math. Soc. 20 (1969), 141-146. MR 38 \#1663.

2. F. E. Browder and W. V. Petryshyn, The solution by iteration of nonlinear functional equations in Banach spaces, Bull. Amer. Math. Soc. 72 (1966), 571-575. MR 32 \#8155b.

3. J. B. Diaz and F. T. Metcalf, On the structure of the set of subsequential limit points of successive approximations, Bull. Amer. Math. Soc. 73 (1967), 516-519. MR 35 \#2268.

4. M. Edelstein, On fixed and periodic points under contractive mappings, J. London Math. Soc. 37 (1962), 74-79. MR 24 \#A2936.

5. S. B. Nadler, Multivalued contraction mappings, Pacific J. Math. 30 (1969), $475-488$.

6. R. E. Smithson, Some general properties of multi-valued functions, Pacific J. Math. 15 (1965), 681-703. MR 32 \#437.

7. - , A note on $M$-isometrics and contractive multifunctions, Notices Amer. Math. Soc. 17 (1970), 222. Abstract \#672-486.

8. W. L. Strother, Continuous multi-valued functions, Bol. Soc. Mat. Saō Paulo 10 (1958), 87-120. MR 23 \#A292.

9. T. Yoshizawa, Stability theory by Liapunov's second method, Publ. Math. Soc. Japan, no. 9, Math. Soc. Japan, Tokyo, 1966. MR 34 \#7896.

UNIVERSITY OF WYoming, LARAMIE, Wyoming 82070 